\title{
Practical application of the Kramers-Kronig transformation on impedance measurements in solid state electrochemistry
}

\author{
Bernard A. Boukamp \\ Department of Chemical Technology, Laboratory for Inorganic Chemistry, Materials Science and Catalysis, University of Twente, \\ P.O. Box 217, 7500 AE Enschede. The Netherlands
}

Received 16 December 1992; accepted for publication 19 March 1993

\begin{abstract}
A method is presented for executing the Kramers-Kronig transforms of electrochemical impedance data on a computer. Attention is paid to the extrapolation techniques for impedance data with a limited frequency range. It is shown that impedance spectra of systems with blocking electrodes, exhibiting a CPE-type response, are also Kramers-Kronig transformable. The necessity and limitations of these transforms with respect to impedance data are briefly discussed.
\end{abstract}

\section{Introduction}

Frequency dependent impedance measurements (or electrochemical impedance spectroscopy, EIS for short) have become an important research tool in electrochemistry. Especially since the availability of powerful complex nonlinear least squares (CNLS) data analysis programs (e.g. LOMPF and LEVM, written by Macdonald [1-3], and EQUIVALENT CIRCUIT by the author $[4,5]$ ), the use of impedance techniques has increased considerably as these programs allow the quantitative analysis of complicated dispersion data.

For the analysis it is important to have an adequate model function, often presented in the form of an equivalent circuit, as well as a set of starting values for the adjustable parameters. The program EQUIVALENT CIRCUIT comprises a simple preanalysis procedure based on the fitting of simple circuits to selected parts of the total dispersion curve, followed by subtraction of these sub-circuit dispersions from the total dispersion [6]. This method, when applied judiciously, can yield both the model function and starting values for the subsequent total CNLS-fit procedure. Examples of this method have been presented in $[7,8]$.

For the equivalent circuit a number of basic dispersion relations or transfer functions (elements) are available $[4,9]$. Especially the Macdonald program
LEVM [2,3] recognizes a number of special dielectric dispersion relations $[1,10]$. It is quite possible, however, that no acceptable match can be found between the measured data set, $Z_{i}=Z_{\mathrm{re}, i}+\mathrm{j} Z_{\mathrm{im}, i}$ $(i=1 \ldots N)$, and the model dispersion function, $Z\left(\omega_{i}\right)=Z_{\mathrm{re}}\left(\omega_{\mathrm{i}}\right)+\mathrm{j} Z_{\mathrm{im}}\left(\omega_{i}\right)$. A discrepancy in the fit is most clearly observed in a plot of the relative residuals of the fit result

$$
\begin{aligned}
& \Delta_{\mathrm{re}, i}=\frac{Z_{\mathrm{re}, i}-Z_{\mathrm{re}}\left(\omega_{i}\right)}{\left|Z\left(\omega_{i}\right)\right|}, \\
& \Delta_{\mathrm{im}, i}=\frac{Z_{\mathrm{im}, i}-Z_{\mathrm{im}}\left(\omega_{i}\right)}{\left|Z\left(\omega_{i}\right)\right|},
\end{aligned}
$$

versus the logarithm of the frequency [11]. For a correct CNLS-fit these residuals should scatter randomly around the abscissa. An inappropriate fitting function will lead to a systematic distribution of the residuals, i.e. they form a distinct trace with respect to the abscissa. Several causes may be responsible for the incorrect fit result:

(i) systematic errors in the data file due to a change in the electrochemical system (e.g. drifting temperature, aging, reaction) or due to a distortion caused by the measurement system;

(ii) use of an inappropriate equivalent circuit;

(iii) the electrochemical system exhibits a nonideal transfer function, i.e. it cannot be represented by a set of simple elementary transfer functions. 
In principle there is no real difference between case (ii) and case (iii), but it is useful to make a distinction between using an incorrect equivalent circuit while a correct one can be constructed or that no simple equivalent circuit (within the bounds of the data analysis program) can be found. It is of importance to be able to discern between case (i) and cases (ii) and (iii), i.e. between distorted data, and correct data but modelled with an improper equivalent circuit.

This distinction can be made with the help of the Kramers-Kronig (K-K) relations [12]. Correct impedance (and admittance) dispersions must obey these Kramers-Kronig relations, which relate the real and imaginary parts of the impedance spectrum to each other. In short, the imaginary value of each data point can be obtained through a special transform of the complete (from $\omega=0$ to $\infty$ ) real part of the impedance spectrum. Similarly the real value of each data point, apart from a constant, can be obtained by a different transform of the complete imaginary part of the impedance spectrum. The conditions necessary for a system to comply with the $\mathrm{K}-\mathrm{K}$ relations are generally stated as $[13,14]$ :

(i) causality: the response must only be related to the applied perturbation.

(ii) linearity: only the first order response must be present in the impedance. For nonlinear systems, such as electrodes, this implies the use of small perturbation voltages $\left(<10 \mathrm{mV}_{\mathrm{pp}}\right)$.

(iii) stability: the system may not change with time, nor continue to oscillate when the perturbation is removed.

(iv) finite for all values of $\omega$, including $\omega \rightarrow 0$ and $\omega \rightarrow \infty$.

Examples of the application of $\mathrm{K}-\mathrm{K}$ transforms have been presented by D.D. Macdonald et al. [13], Urquidi-Macdonald et al. [15,16] and J.R. Macdonald [17]. For the impedance representation of the dispersion the following special Kramers-Kronig relations exist. For the real part of the impedance

$$
Z_{\mathrm{re}}(\omega)=Z_{\mathrm{re}}(\infty)+\frac{2}{\pi} \int_{0}^{\infty} \frac{x Z_{\mathrm{im}}(x)-\omega Z_{\mathrm{im}}(\omega)}{x^{2}-\omega^{2}} \mathrm{~d} x,
$$

or

$$
\begin{aligned}
& Z_{\mathrm{re}}(\omega)=Z_{\mathrm{re}}(0) \\
& +\frac{2 \omega}{\pi} \int_{0}^{\infty} \frac{(\omega / x) Z_{\mathrm{im}}(x)-Z_{\mathrm{im}}(\omega)}{x^{2}-\omega^{2}} \mathrm{~d} x
\end{aligned}
$$

and for the imaginary part

$Z_{\mathrm{im}}(\omega)=\frac{2 \omega}{\pi} \int_{0}^{\infty} \frac{Z_{\mathrm{re}}(x)-Z_{\mathrm{re}}(\omega)}{x^{2}-\omega^{2}} \mathrm{~d} x$.

Correct data must obey these $\mathrm{K}-\mathrm{K}$ relations. Hence by comparing the transformed real part, using eq. (4), with the imaginary part of the impedance spectrum, and vice versa for the imaginary to real transform (eq. (2) or (3)), the validity of the impedance data can be tested. If a discrepancy is observed between the actual and transformed values (within the range of experimental error) then the data must be assumed to be corrupted. There are, however, a few exceptions where the data can be modelled with an equivalent circuit, but fails the $\mathrm{K}-\mathrm{K}$ transform test. This generally occurs when a negative (differential) resistance is present, a condition typically encountered in corrosion studies $[9,14,16]$.

\section{Discrete K-K transform}

Although several publications have been presented on applying the K-K transform [13-17], little information has been given on the procedures. The most simple way is to perform a discrete integration

$$
\int_{x_{1}}^{x_{n}} y_{i} \mathrm{~d} x \simeq \sum_{i=1}^{n-1} \int_{x_{i}}^{x_{i}+1} Y_{i}(x) \mathrm{d} x,
$$

where $y_{i}$ represents the integrand value of eqs. (2)(4) obtained at $x_{i}$, and $Y_{i}(x)$ is an integrable function (e.g. polynomial or cubic spline) which is chosen such that it follows the $y_{i}$ values as closely as possible. In different intervals of $x$ different coefficients for $Y_{i}(x)$ may be used. For the $\mathrm{K}-\mathrm{K}$ transforms (eqs. (2) -(4)) a problem arises for $x_{i}=\omega$, which incorrectly sometimes is referred to as a singularity. Actually, eqs. (2)-(4) are special forms of the K-K transformations in which the singularity has been removed. For a detailed discussion of the $\mathrm{K}-\mathrm{K}$ transforms see ref. [18]. 
There are two ways to deal with the $x=\omega$ problem. The most simple one is to ignore this specific data point and exclude it from the integration procedure, assuming that the integration function will faithfully reproduce the curve at $x_{i}=\omega$. The second option is to evaluate $y_{i}$ at $x_{i}=\omega$, which can be useful if the distance between data points on the frequency scale is rather large (e.g. 4 to 5 points per decade). By developing $Z_{\mathrm{re}}(x)$, or $Z_{\mathrm{im}}(x)$, in a Taylor series around $x=\omega$ one obtains for eq. (2):

$$
\begin{aligned}
& \frac{x Z_{\mathrm{im}}(x)-\omega Z_{\mathrm{im}}(\omega)}{x^{2}-\omega^{2}} \\
& \quad=\left[x Z_{\mathrm{im}}(\omega)+\left.x(x-\omega) \frac{\mathrm{d} Z_{\mathrm{im}}(x)}{\mathrm{d} x}\right|_{x=\omega}\right. \\
& \left.\quad+\left.x \frac{(x-\omega)^{2}}{2 !} \frac{\mathrm{d}^{2} Z_{\mathrm{im}}(x)}{\mathrm{d} x^{2}}\right|_{x=\omega}+(\ldots)-\omega Z_{\mathrm{im}}(\omega)\right] \\
& \quad \times[(x-\omega)(x+\omega)]^{-1},
\end{aligned}
$$

which, for $x \rightarrow \omega$, simplifies to

$$
\begin{aligned}
& \lim _{x \rightarrow \omega} \frac{x Z_{\mathrm{im}}(x)-\omega Z_{\mathrm{im}}(\omega)}{x^{2}-\omega^{2}} \\
& \quad=\frac{1}{2}\left[\frac{Z_{\mathrm{im}}(\omega)}{\omega}+\left.\frac{\mathrm{d} Z_{\mathrm{im}}(x)}{\mathrm{d} x}\right|_{x=\omega}\right] .
\end{aligned}
$$

This transformation provides a more rigorous proof for the finiteness of $y_{i}$ at $x=\omega$ than the binomial expansion presented in ref. [16]. Similarly the integrand of eq. (4) can be expressed for $x=\omega$ by

$$
\lim _{x \rightarrow \omega} \frac{Z_{\mathrm{re}}(x)-Z_{\mathrm{re}}(\omega)}{x^{2}-\omega^{2}}=\left.\frac{1}{2 \omega} \frac{\mathrm{d} Z_{\mathrm{re}}(x)}{\mathrm{d} x}\right|_{x=\omega} .
$$

Thus if the derivatives of the real and imaginary components are available then the special point $x=\omega$ can be included in the integration procedure.

\section{Derivatives}

The derivatives of the real and imaginary components are not directly available. They can be approximated by fitting the real and imaginary parts of the impedance with a polynomial expression. As the data most likely will contain some noise (statistical errors) some degree of smoothing should be applied. This can be accomplished by fitting a polynomial of degree $m$ ( $m+1$ adjustable parameters)

$$
Y(\omega)=\sum_{i=0}^{m} a_{i} \omega^{i},
$$

to a set of $p$ sequential data points $(p>m)$, using a least squares fit procedure:

$$
\begin{aligned}
& \frac{\mathrm{d}}{\mathrm{d} a_{i}} \sum_{j=k}^{k+p-1}\left(y_{j}-Y\left(\omega_{j}\right)\right)^{2}=0, \\
& \quad i=0, \ldots, m,
\end{aligned}
$$

where $y_{j}$ represents the real (or imaginary) part of the data and $k$ to $k+p-1$ the range of $p$ data points in the data set to be fitted. The derivatives (in that range) can be found directly:

$$
\frac{\mathrm{d}}{\mathrm{d} \omega} Y(\omega)=\sum_{i=1}^{m} i a_{i} \omega^{i-1} \text {. }
$$

\section{Example}

In order to demonstrate the applicability of the discrete Kramers-Kronig transforms synthetic impedance data (fig. 1) was generated using a simple equivalent circuit (insert fig. 1) consisting of two dispersive sub-circuits (parallel resistance and capacitance, and parallel resistance and Warburg) in series with a resistor. Parameter values are tabulated in table 1 . The frequency ranged from $1 \mathrm{mHz}$ to 10 $\mathrm{MHz}$ at 7 frequencies per decade. The $\mathrm{K}-\mathrm{K}$ transforms were calculated in the range $0.1 \mathrm{~Hz}$ to $0.1 \mathrm{MHz}$.

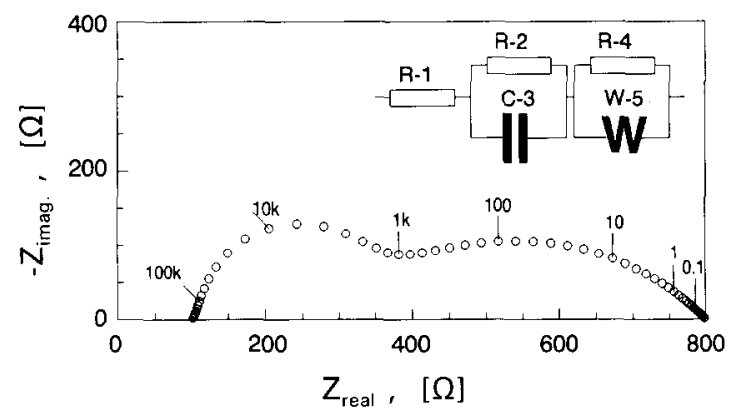

Fig. 1. Impedance spectrum of the test circuit presented in the insert of this figure. Circuit parameter values are given in table 1. The frequency range is $1 \mathrm{mHz}-10 \mathrm{MHz}$. The $\mathrm{K}-\mathrm{K}$ transform values are calculated for the range $0.1 \mathrm{~Hz}-100 \mathrm{kHz}$. Frequencies are in $\mathrm{Hz}$. 
Table 1

Parameter values of the equivalent circuit used for the example dispersion presented in fig. 1 .

\begin{tabular}{|c|c|c|}
\hline Element & & Parameter value \\
\hline $\mathrm{R}_{1}$ & & $100 \Omega$ \\
\hline $\mathrm{R}_{2}$ & & $200 \Omega$ \\
\hline $\mathrm{C}_{3}$ & & $1 \times 10^{-7} \mathrm{~F}$ \\
\hline $\mathrm{R}_{4}$ & & $500 \Omega$ \\
\hline $\mathbf{W}_{5}$ & {$\left[Y_{0}(\mathrm{j} \omega)^{0.5}\right]$} & $1 \times 10^{-4} S \cdot s^{-0.5}$ \\
\hline
\end{tabular}

The low end and high end frequencies were used to extend the integration range. Besides the exact data set also two data sets with added noise $(0.2 \%$ and $0.5 \%$ relative to $|Z(\omega)|)$ were generated over the frequency range $0.1 \mathrm{~Hz}$ to $0.1 \mathrm{MHz}$ using the CNLS program EQUIVALENT CIRCUIT [5].

As the equivalent circuit is rather simple the exact derivatives could be calculated, so that a direct comparison could be made with the derivative estimates obtained through the polynomial LS-fit procedure. For the noise free impedance data set a series of derivatives were calculated using different combinations of $p$ (data sub-set length) and $m$ (polynomial degree). The absolute values of the relative deviations, $\Delta_{\text {diff }}$,

$A_{\mathrm{diff}}=\frac{\mathrm{d} Z_{j}(\omega) / \mathrm{d} \omega-\mathrm{d} Y(\omega) / \mathrm{d} \omega}{\mathrm{d} Z_{j}(\omega) / \mathrm{d} \omega}$,

$$
j=\text { re or im, }
$$

with respect to the exact imaginary differential values are presented in fig. 2 for the imaginary part of the impedance. Here $Y(\omega)$ represents the polynomial function fitted to the real or imaginary part of the dispersion (eqs. (9), (10)). For clarity the exact imaginary differential is also shown in fig. 2. In order to limit the range the exact differential is presented in the form: $\omega \cdot \mathrm{d} Z_{\mathrm{im}}(\omega) / \mathrm{d} \omega$. For the real part of the spectrum presented in fig. 1 identical results were obtained.

For the evaluation of the coefficients, $a_{i}$, of the polynomial expression a Gaussian elimination/backsubstitution algorithm [19] was used to solve the set of simultaneous equations represented by eq. (10). In the polynomial differentiation $\ln \omega$ was used as variable as this produced better results than the use of $\omega$.

From the results presented in fig. 2 it is obvious

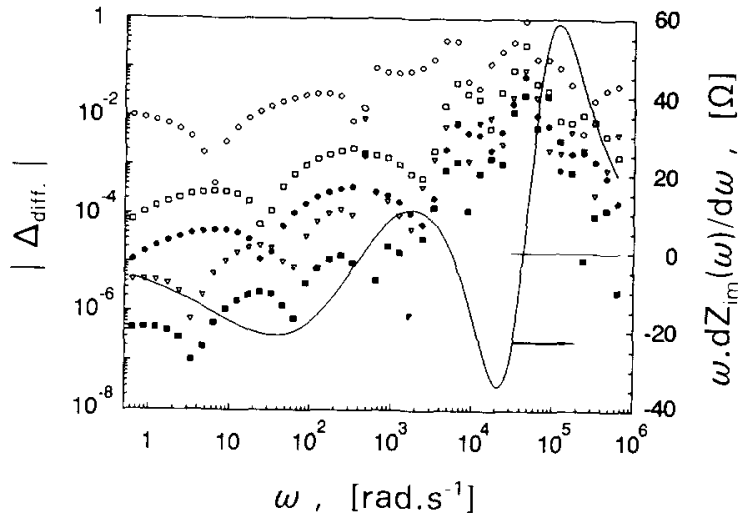

Fig. 2. Absolute values of the relative deviation, $A_{\text {diff, }}$, of the estimated differentials of $Z_{\mathrm{im}}(\omega)$ with respect to the exact differentials for several combinations of the data sub-range, $p$, and the polynomial degree, $m:(\bigcirc) p=5, m=2 ;(\odot) p=5, m=4 ;(\square)$ $p=7, m=4$, (目) $p=7, m=6 ;(\nabla) p=9, m=6$. The modified exact differential, $\omega \mathrm{d} Z_{\mathrm{im}}(\omega) / \mathrm{d} \omega$ (drawn line), is shown for clarity.

that with a higher degree polynomial (with $p=m+1$ ) a more accurate value for the differential is obtained. Applying some degree of smoothing, e.g. by adding two more points to the data set $(p=m+3)$, decreases the accuracy for this noise free data set. For $p=m+1$ an exact solution for the polynomial is obtained, but the derived differential value not necessarily equals the exact value.

In fig. 3 the absolute values of the relative deviation of the derivatives, with respect to the exact values, of the imaginary component are presented for the $0.2 \%$ and $0.5 \%$ noise added example dispersions generated with the equivalent circuit of fig. 1. Analysis showed that with a sub-set size of $p=9$ an optimum was obtained for $m=3$ or 4 . Fig. 3 presents the case for $m=4$. The optimum amount of smoothing, however, may strongly depend on the form of the impedance spectrum. The errors in the estimated derivatives are much larger than the errors in the data set. Hence for data with appreciable noise this method is not well suited for establishing the integrand value at $x^{2}=\omega^{2}$ in the transform integrals.

The integrands of the $\mathrm{K}-\mathrm{K}$ transforms, however, are well-behaved functions, as is demonstrated in fig. 4. Here the integrands of the real (eq. (4)) and imaginary (eq. (2)) transforms, $y_{\mathrm{re}}(x, \omega)$ and $\omega \cdot y_{\mathrm{im}}(x$, $\omega)$ are presented as function of $x$ for three values of $\omega: 2 \pi, 2 \pi \times 10^{2}$ and $2 \pi \times 10^{4} \mathrm{rad} \mathrm{s}^{-1}$. Arrows mark 


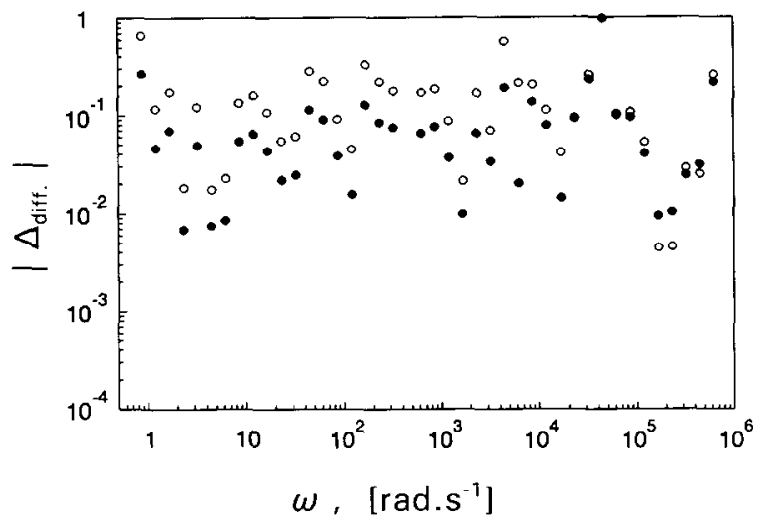

Fig. 3. Absolute value of the relative deviation of the estimated differentials of $Z_{\mathrm{im}}(\omega)$ with respect to the exact differentials, $\Delta_{\mathrm{diff}}$ for noisy data with $(O) 0.2 \%$, and $(O) 0.5 \%$ relative noise added. Data sub-set range, $p=9$, polynomial degree, $m=6$. Data was generated using the equivalent circuit of fig. 1 and parameter values of table 1 .

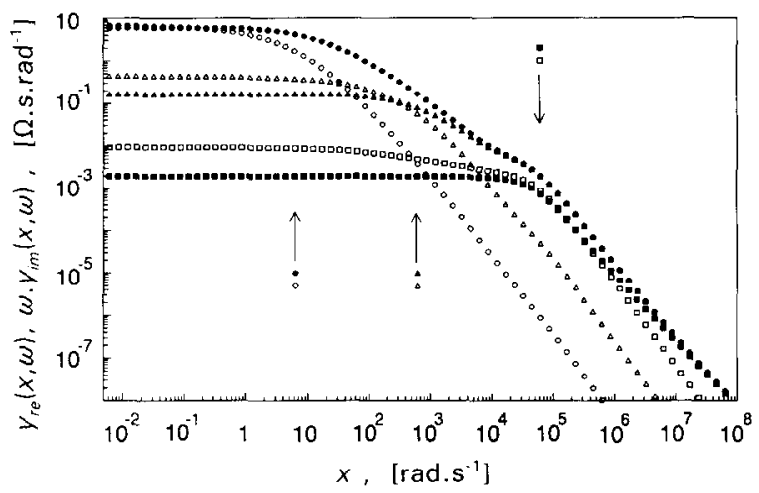

Fig. 4. Integrands for the real to imaginary $\mathrm{K}-\mathrm{K}$ transform, $y_{\mathrm{re}}(x$, $\omega)$ (closed symbols), and for the imaginary to real transform, $\omega y_{\mathrm{re}}(x, \omega)$ (open symbols), as function of $x$ for three values of $\omega:(\bullet, O) \omega=2 \pi,(\boldsymbol{\Lambda}, \Delta) \omega=2 \pi \times 10^{2}$ and $(\boldsymbol{\square}, \square) \omega=2 \pi \times 10^{4}$ rad s$~^{-1}$. The three $\omega$ positions are indicated by arrows.

the $x=\omega$ positions, the corresponding data points were calculated using eqs. (7) and (8).

If the spacing of the data points on the $\omega$-axis is close enough then the special point $x=\omega$ may be omitted from the discrete integration procedure as proposed above, assuming that the modelling function (cubic spline or polynomial) will closely reproduce the omitted data point. For highly accurate data (little noise) the derivative estimation method may be used. In the following procedures the simple method, i.e. skipping the point $x=\omega$, will be followed.

\section{Discrete transform procedure}

Two routes to perform the discrete transform, i.e. the discrete integration, will be presented here. The first and fastest route is by creating a cubic spline function through the data sets $\left[x_{j}, y_{j}\right]$. The procedure is well documented in many textbooks on numerical computer algorithms, c.g. ref. [19]. The cubic spline function, which is continuous in its first derivative, can then be presented by:

$$
\begin{aligned}
& y(x)=A y_{j}+B y_{j+1}+\left(\left(A^{3}-A\right) Y_{j}^{\prime \prime}+\left(B^{3}-B\right) Y_{j+1}^{\prime \prime}\right) \\
& \quad \times\left(x_{j+1}-x_{j}\right)^{2} / 6
\end{aligned}
$$

with

$A=\frac{x_{j+1}-x}{x_{j+1}-x_{j}}$ and $B=\frac{x-x_{j}}{x_{j+1}-x_{j}}$.

The $Y_{j}^{\prime \prime}$ values represent the second derivatives of the interpolating polynomial. A table of these values is obtained through a standard subroutine (e.g. [19]). The importance of eq. (13) is that an analytic expression can be derived for the integral of the cubic spline function:

$$
\begin{gathered}
\int_{x_{j}}^{x_{j+1}} y(x) \mathrm{d} x=\frac{1}{2}\left(x_{j+1}-x_{j}\right)\left(y_{j}+y_{j+1}\right) \\
-\left(x_{j+1}-x_{j}\right)^{3}\left(Y_{j}^{\prime \prime}+Y_{j+1}^{\prime \prime}\right) / 24 .
\end{gathered}
$$

Hence the integral can be replaced by a summation over all intervals $x_{j}$ to $x_{j+1}$.

The second route is by fitting a polynomial of degree $m$ through a sub-set of $p$ data points (see above). For the integral of the polynomial also an analytic expression is available

$$
\int_{x_{j}}^{x_{j+p}-1}\left[\sum_{i=0}^{m} a_{i} x^{i}\right] \mathrm{d} x=\sum_{i=0}^{m} \frac{a_{i}}{i+1}\left(x_{j+p-1}^{i+1}-x_{j}^{i+1}\right) .
$$

Thus the data set must be divided in a series of subsets (each end member must also be the first member of the next sub-set), which then can be evaluated individually. The polynomial evaluation is almost a factor 6 slower than the cubic spline evaluation, 
however, once again it may provide some degree of smoothing for noisy data $(p>m+1)$. The smoothing must be used with care as too much smoothing may result in a large deviation from the "hypothetical" noise free curve. One should realize however that the integration, which essentially is a summation, is much less sensitive to noise in the data than the estimation of the derivatives is (see above).

In order to improve accuracy the integration was performed with $\ln x$ instead of $x$, using the relation:

$\mathrm{d} x=x \mathrm{~d} \ln x$.

The value of $Z_{\text {re }}(\infty)$ in eq. (2) was obtained by calculating the mean difference between the real part of the actual impedance and the $\mathrm{K}-\mathrm{K}$ transform of the imaginary part.

First the $\mathrm{K}-\mathrm{K}$ transforms were calculated with the integration range from $x=2 \pi \times 10^{-1}$ to $2 \pi \times 10^{5} \mathrm{rad}$ $\mathrm{s}^{-1}(0.1 \mathrm{~Hz}$ to $0.1 \mathrm{MHz})$ using the exact data set presented in fig. 1 . The relative deviations between the $\mathrm{K}-\mathrm{K}$ transform (spline method) and the actual data set, $A_{\mathrm{KKre}}$ and $\Delta_{\mathrm{KKim}}$, are presented in fig. 5 (circles). The end regions of the real to imaginary transform (graph symbol $O$ ) show a large deviation due to the limited range of the integration. The $\mathrm{K}-\mathrm{K}$ transforms calculated with the polynomial expressions gave identical results for all combinations of $p$ and $m$ values used in the evaluation of the derivatives (fig. 2).

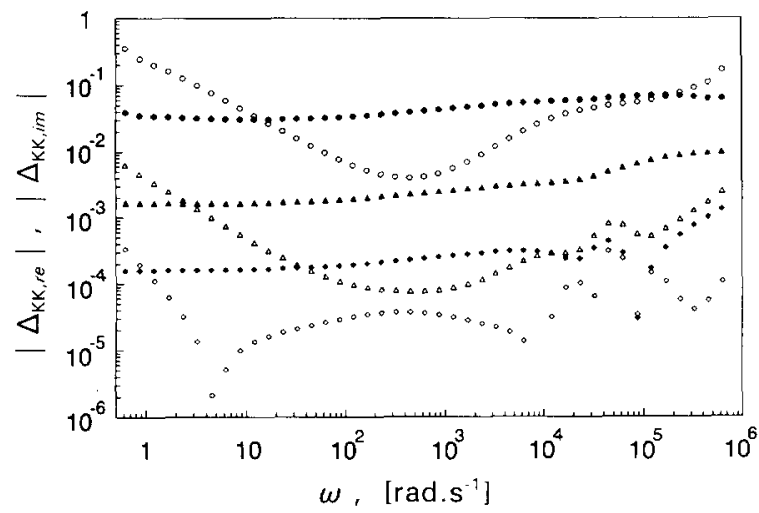

Fig. 5. Plot of the absolute values of the relative differences, $\Delta_{\mathrm{re}}$ and $\Delta_{\text {im }}$, between the exact data of fig. 1 and the K-K transforms: $(\bullet, O)$ using the limited range of $0.1 \mathrm{~Hz}-0.1 \mathrm{MHz} ;(\Delta, \Delta)$ using two decades extrapolation at the low and the high frequency side (eqs. (18) and (19)), and $(\diamond, \diamond)$ with extrapolation to $x=0$ and $x=\infty$ (+eqs. (21) and (22)). Open symbols for the real to imaginary transform, closed symbols for the reverse transforms. A spline integration procedure was used.
A substantial improvement in the transforms can be obtained by extrapolation of the impedance spectrum over several orders in frequency (variable $x$ ) while restricting the $\mathrm{K}-\mathrm{K}$ transform to the measured frequency range (variable $\omega$ in eqs. (2)-(4)). The triangles in fig. 5 represent the relative deviations of the $\mathrm{K}-\mathrm{K}$ transforms with an integration range of $x_{\min }=\omega_{\min } \times 10^{-2}$ and $x_{\max }=\omega_{\max } \times 10^{2}(1 \mathrm{mHz}$ to $10 \mathrm{MHz}$ ), using the complete exact data set of fig. 1.

For actual impedance measurements this extended data set must be created by extrapolation of the end ranges. This is conveniently done with a "partial data fit". The accuracy of the extrapolation strongly depends on the amount of noise in the dispersion data. Moreover it is important that the dispersion measurement extends into a region that may be modelled with a single time constant, e.g. a (depressed) semi-circle. If there is a significant influence of a second dispersive process then the extrapolation will be strongly flawed.

The improvement in the $\mathrm{K}-\mathrm{K}$ transforms can be enhanced by using an even larger extrapolation range (e.g. 4 decades on both sides), but this will drastically increase the calculation time. Considering the shape of the integrands in fig. 4 it is clear that for $x \ll \omega$ or $x \gg \omega$ the integrand function in the log-log plot can be approximated by a straight line:

$\ln y=C(\ln x)+D$, for $x \ll \omega$.

Thus the last missing regions in the integration can be obtained by fitting lines according to eq. (17) to the end regions, which can then be integrated analytically. With $\ln x$ as integration variable this results in

$\int_{-\infty}^{\ln x_{\min }} \mathrm{e}^{C \ln x+D} \mathrm{~d} \ln x=\mathrm{e}^{D} x_{\min }^{C} / C$,

and

$$
\int_{\ln x_{\max }}^{\infty} \mathrm{e}^{C \ln x+D} \mathrm{~d} \ln x=-\mathrm{e}^{D} x_{\max }^{C} / C,
$$

provided that $C$ is positive in eq. (18) and negative in eq.: (19). Including this final extrapolation to $x=0$ and $x=\infty$ a further improvement in the $\mathrm{K}-\mathrm{K}$ transforms is obtained. This is clearly demonstrated in fig. 5 by the relative deviations (diamonds) for the 
complete $\mathrm{K}-\mathrm{K}$ transforms, including eqs. (18) and (19), of the exact data set of fig. 1 . If the deviations are taken relative to the modulus of the impedance vector (eq. (1)) even lower values, especially for the real to imaginary transform, are obtained for the $\Delta_{\mathrm{KK}}$.

An example of the complete $\mathrm{K}-\mathrm{K}$ transforms of the impedance data of fig. 1 , but containing $0.2 \%$ and $0.5 \%$ noise (relative to the modulus of the impedance vector), is presented in fig. 6 . The scatter in the relative deviations, $A_{\mathrm{KKre}}$ and $\Delta_{\mathrm{KKim}}$, of the $\mathrm{K}-\mathrm{K}$ transforms appears larger than in the original data, but when the deviations are taken relative to the modulus of the impedance vector (eq. (1)) the scatter becomes comparable to the original noise in the data set.

The full range $\mathrm{K}-\mathrm{K}$ transforms of the data set of fig. 1 (43 frequencies), using the spline integration routine, took less than $2 \mathrm{~s}$ on a $25 \mathrm{MHz} 80386$ personal computer with coprocessor ( $80387 \mathrm{chip})$. The
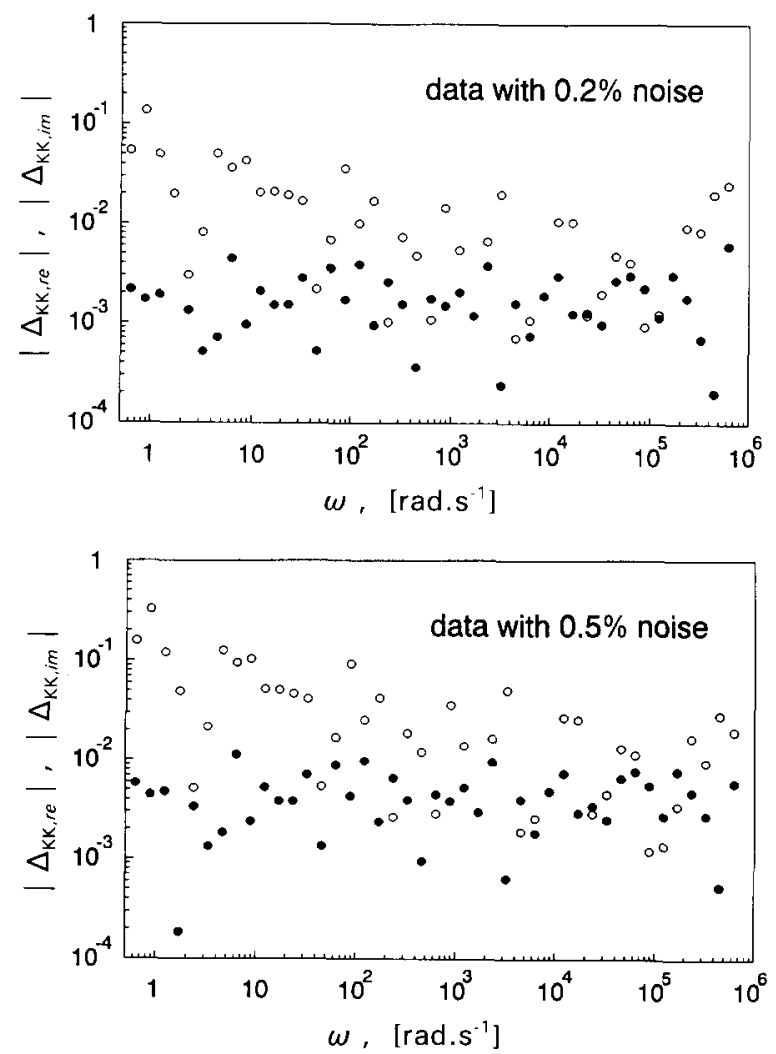

Fig. 6. Plot of the absolute values of the relative differences, $\Delta_{\mathrm{KKre}}$ (O) and $\Delta_{\text {KKim }}(O)$ for the impedance data of fig. 1, but polluted with $0.2 \%$ (upper graph) and $0.5 \%$ (lower graph) noise. polynomial routine, with sub-set length $p=9$ and polynomial degree $m=6$, took $10.5 \mathrm{~s}$ for the same data set. The results obtained by both procedures, however, were very close. The $\mathrm{K}-\mathrm{K}$ test program used was written and compiled in Turbo Pascal v. 6.0 (trademark Borland International Inc.) \#1 using double precision for the floating point variables.

\section{Blocking electrodes}

The Kramers-Kronig rules require the data to be finite for $\omega \rightarrow 0$ and $\omega \rightarrow \infty$. For impedance measurements on ionically conducting solid samples with blocking electrodes the condition of finiteness for $\omega \rightarrow 0$ is seldom met. Generally an "open ended" CPE (constant phase element) type dispersion is observed for $\omega \rightarrow \omega_{\min }$, where $\omega_{\min }$ represents the lowest frequency measured. As each system will have a dc conductance, however small it may be, the low frequency dispersion will eventually return to the real axis for $\omega \rightarrow 0$. But this will occur at extremely low frequencies for which it is impractical (if not impossible) to carry out reliable impedance measurements. Fortunately the dispersion relation of a CPE element (impedance representation: $Z(\omega)=$ $\left.Z_{0}(j \omega)^{-\alpha}\right)$ does obey the K-K transforms [20] resulting in a finite value for the transform integral. This can be demonstrated by considering the transform integral for a CPE element in the range $0 \leqslant$ $x \leqslant x_{\min }$, with $x_{\min } \ll \omega$. With $x^{2} \ll \omega^{2}$ the integral of eq. (2) simplifies to

$$
\begin{aligned}
& \int_{0}^{x_{\min }} \frac{x Z_{0, \mathrm{im}} x^{-\alpha}-\omega Z_{0, \mathrm{im}} \omega^{-\alpha}}{x^{2}-\omega^{2}} \mathrm{~d} x \\
& \approx \frac{Z_{0, \mathrm{im}}}{\omega^{2}} \int_{0}^{x_{\min }} \omega^{1-\alpha}-x^{1-\alpha} \mathrm{d} x \\
& =Z_{0, \mathrm{im}}\left[\frac{\omega^{1-\alpha} x_{\min }}{\omega^{2}}-\frac{x_{\min }^{2-\alpha}}{\omega^{2}(2-\alpha)}\right],
\end{aligned}
$$

with

\#1 Most of the algorithms described in this paper are available from standard computer libraries. However, interested readers may obtain the source code of the described procedures by sending a formatted diskette to the author in a reusable mailer. 
$Z_{0, \mathrm{im}}=Z_{0} \sin \frac{\alpha \pi}{2}$

yielding a finite value. As the integral for $x_{\min } \leqslant x \leqslant \infty$ also will yield a finite value the entire $K-K$ transform remains finite. Similarly the imaginary to real transform will yield a finite value.

This can be used to perform the "end region extrapolation", in analogy with the regular case discussed above, for the blocking electrode. If the low frequency end of the measurement is in a single time constant range then it can be extrapolated over a few decades using a resistance, $R_{\mathrm{CPE}}$, in series with $\mathrm{CPE}$. While the regular transforms (eqs. (2) and (4)) are carried out over the extended frequency range, the low frequency end-correction, for $0 \leqslant x \leqslant x_{\min }$ with $x \ll \omega$, can be found from

$$
\begin{aligned}
& \int_{0}^{x_{\min }} \frac{x Z_{0, \mathrm{im}} x^{-\alpha}-\omega Z_{\mathrm{im}}(\omega)}{x^{2}-\omega^{2}} \mathrm{~d} x \\
& \approx-\frac{1}{\omega^{2}} \int_{0}^{x_{\min }} Z_{0, \mathrm{im}} x^{1-\alpha}-\omega Z_{\mathrm{im}}(\omega) \mathrm{d} x \\
& =\frac{x_{\min }}{\omega} Z_{\mathrm{im}}(\omega)-\frac{x_{\min }^{2-\alpha}}{\omega^{2}(2-\alpha)} Z_{0, \mathrm{im}},
\end{aligned}
$$

with

$Z_{0, \mathrm{im}}=Z_{0} \sin \frac{\alpha \pi}{2}$

Similarly the value of the integral of eq. (4) in the range $0 \leqslant x \leqslant x_{\min }$, with $x_{\min } \ll \omega$, is found:

$$
\begin{aligned}
& \int_{0}^{x_{\min }} \frac{R_{\mathrm{CPE}}+Z_{0, \mathrm{re}} x^{-\alpha}-Z_{\mathrm{im}}}{x^{2}-\omega^{2}} \mathrm{~d} x \\
& \quad \approx \frac{x_{\min }}{\omega^{2}}\left(Z_{\mathrm{re}}(\omega)-R_{\mathrm{CPE}}\right)-\frac{x_{\min }^{1-\alpha}}{(1-\alpha) \omega^{2}} Z_{0, \mathrm{re}}
\end{aligned}
$$

with

$$
Z_{0, \text { re }}=Z_{0} \cos \frac{\alpha \pi}{2}
$$

Hence in the case of blocking electrodes with clear CPE behaviour at low frequencies the following procedure can be applied for evaluation of the K-K transforms:

(i) Extrapolate the measured impedance to a lower frequency limit, $\omega_{\text {ext }}, 10^{-2}$ times the lowest mea- sured frequency (thus $x^{2} \ll \omega^{2}$ ) by fitting a series combination of a resistance and a CPE using a CNLS type procedure $[5,6]$.

(ii) Calculate the transform integrals in the range $\omega_{\text {ext }}$ to $\infty v$ ng either the spline or polynomial integration a id the high frequency end-point correction of eq (19).

(iii) Calculate the low frequency end-point correction using eq. (21) or (22). To demonstrate this procedure a new set of synthetic data, with $0.2 \%$ relative noise added, was produced using the program EQUIVALENT CIRCUIT. The equivalent circuit, consisting of a parallel $\mathrm{R}-\mathrm{C}$ circuit in series with a CPE $(\alpha=0.7)$, and the impedance dispersion are presented in fig. 7. The circuit parameter values are given in table 2 . The limited data set was extrapolated by two decades at both the low and high fre-

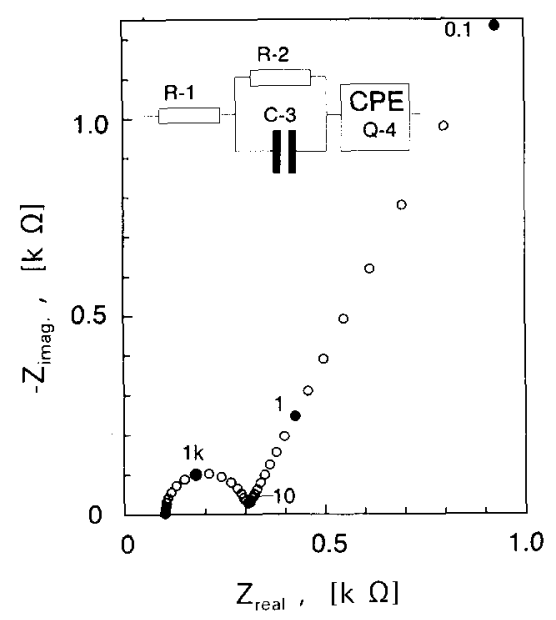

Fig. 7. Impedance spectrum of circuit with "open ended" CPEdispersion at low frequencies, obtained with the equivalent circuit presented in the insert. Parameter values are presented in table 2 . The data was generated over the frequency range $0.1 \mathrm{~Hz}$ to $0.1 \mathrm{MHz}$ and polluted with $0.2 \%$ relative noise using the program EQUIVALENT CIRCUIT.

Table 2

Parameter values of the equivalent circuit used for the example dispersion presented in fig. 7 .

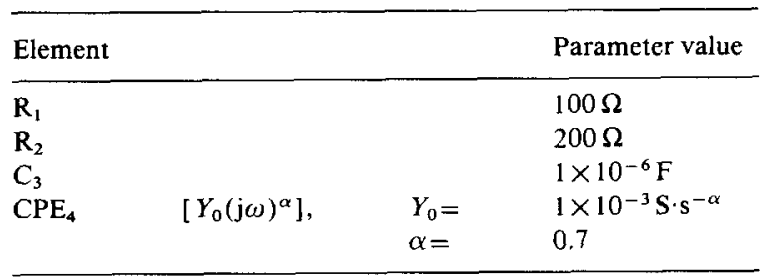


quency end. The low-end extrapolation was modelled with a series combination of a resistance and a CPE, the high frequency end by a (depressed) semicircle which was very close to capacitive behaviour $(\alpha=0.99)$. Parameters were obtained with the CNLS program EQUIVALENT CIRCUIT. The comparison of the actual values and the $\mathrm{K}-\mathrm{K}$ transforms are presented in fig. 8 . The corresponding deviations from the original data set, now taken relative to the modulus of the impedance vector, are presented in fig. 9. These results show that the proposed procedure yields a suitable $K-K$ transform method for blocking electrode measurements.

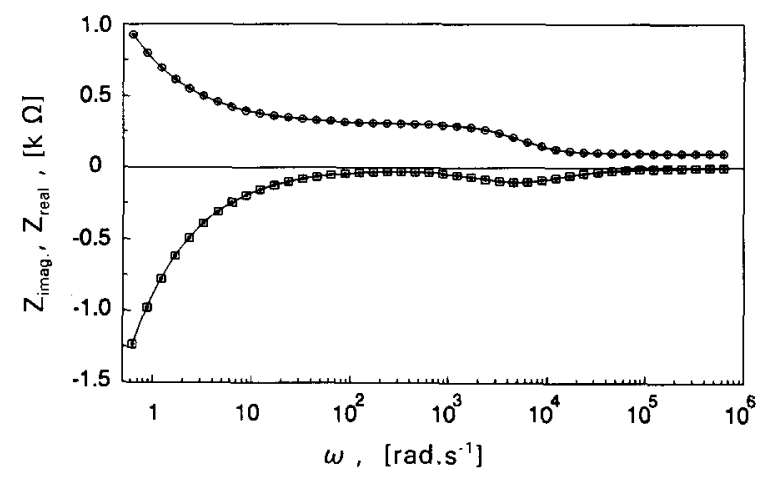

Fig. 8. Comparison fo the K-K transforms (real: $(O)$, imag.: (D)) and actual data set (drawn lines) of fig. 7. The transforms were carried out with the special extrapolations of eqs. (19), (22) and (23) using the spline integration routine.

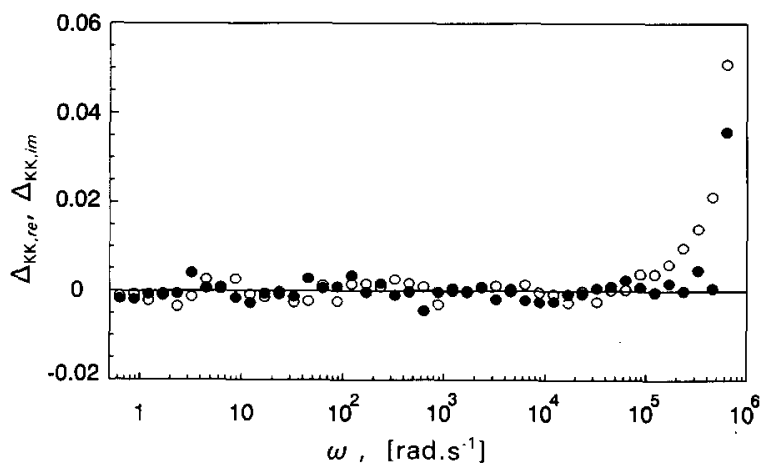

Fig. 9. Plot of the absolute values of the relative differences, $\Delta_{\mathrm{KKre}}$ (O) and $\Delta_{\mathrm{KKim}}(\mathrm{O})$ between the $\mathrm{K}-\mathrm{K}$ transforms and the actual impedance data of fig. 7 . Here the differences are taken relative to the modulus of the impedance vector.

\section{High frequency inductive effects}

Sometimes inductive effects appear in the impedance spectra at high frequency. This is almost always due to contributed lead inductances and/or instrumental distortions. Specifically the current-voltage converters in many potentiostats add a substantial (series) inductive dispersion to the measured impedance at high frequencies. In the impedance representation the imaginary part, which contains the series inductance dispersion, does not transform to yield the real part. This is most clearly demonstrated by taking the imaginary to real transform integral in the range $x_{\max } \leqslant x \leqslant \infty$, with $\omega \ll x_{\max }$

$$
\begin{aligned}
& \int_{x_{\max }}^{\infty} \frac{x L x-\omega L \omega}{x^{2}-\omega^{2}} \mathrm{~d} x \approx \int_{x_{\max }}^{\infty} L-\frac{\omega^{2}}{x^{2}} L \mathrm{~d} x \\
& =L\left[x+\frac{\omega^{2}}{x}\right]_{x_{\max }}^{\infty}=\infty,
\end{aligned}
$$

where $L$ is the value (in Henry) of the apparent series inductance. The failure to transform can simply be understood by considering a simple RL series circuit. In the impedance representation the real part does not contain any information on the dispersive component (the inductance), hence the imaginary part cannot be derived from the real part. Thus these "high frequency" inductive effects must be removed from the measured dispersion before a $\mathrm{K}-\mathrm{K}$ transform, in the impedance representation, can be applied. This is a rather simple procedure as these inductive effects can be accurately measured using a known resistance in place of a sample. As the inductive effect is generally added in series to the impedance dispersion subtraction is straightforward. In the admittance representation, however, the $\mathrm{K}-\mathrm{K}$ transform can be applied to a system containing a series inductance.

\section{Discussion}

In practical applications the use of the $\mathrm{K}-\mathrm{K}$ transforms seems to be limited by two shortcomings of real data: (i) a limited, finite frequency range, and (ii) the presence of noise. With respect to the first restriction, this strongly depends on the type of elec- 
trochemical system under investigation. In some cases the limit values for the impedance, $Z(\omega=0)$ and $Z(\omega=\infty)$, are readily approached in a normal range of frequencies (e.g. $1 \mathrm{mHz}-10 \mathrm{MHz}$ ). The $\mathrm{K}-$ $\mathrm{K}$ transformations can then be calculated with little error in the values for the end ranges. But generally this is not the case and an "end region extrapolation" must be included. If the dispersion in the end region is mainly due to one dispersive process, then extrapolation can simply be done by using a depressed semicircle model or, at low frequencies for blocking electrodes, a single CPE/resistance model (eqs. (21) and (22)). If the end region of the dispersion also contains a significant contribution of a second dispersive process then this must be included in the extrapolation. Simple estimations of the necessary parameters for the extrapolation model will then be more difficult and less precise. Partial CNLSfit analysis of the end regions of the impedance spectrum may be used to elucidate the proper dispersion model. The interactive CNLS program EQUIVALENT CIRCUIT can be helpful in this respect through its pre-analysis sub-program [6] combined with the data simulation sub-program [5].

The presence of noise is mostly a problem with respect to the estimation of the end regions extrapolation. Testing various kinds of simulated data that was polluted with random noise, relative to the impedance vector modulus, showed that the noise level in the $\mathrm{K}-\mathrm{K}$ transformed data was somewhat smaller than in the original data. Hence if the systematic ("non $\mathrm{K}-\mathrm{K}$ ") error in the data is larger than the mean statistical error, it will also appear in the comparison of the real data set and its K-K transform, provided that the end region extrapolations do not contribute a significant error to the transforms. A study on the propagation of errors in the $\mathrm{K}-\mathrm{K}$ transforms is important in this respect. A brief discussion on this subject has recently been presented by Macdonald [17].

A serious problem arises when it is clear that one of the end regions of the measured dispersion cannot be modelled with the available dispersion relations. This means that no proper end region extrapolation can be carried out while this extrapolation is especially crucial to the $\mathrm{K}-\mathrm{K}$ transforms of the end region data points. In this case it is essential to extend the frequency range of the measurement as far as possible so that the corresponding impedances approach the limit value.

Another solution to this problem may be the extrapolation method recently presented by Orazem et al. [21,22]. In their approach the missing low frequency part is modelled with two sets of polynomials, one for the real part and one for the imaginary part of the dispersion. On the polynomials the restriction is imposed that, together with the measured data set, they must obey the $\mathrm{K}-\mathrm{K}$ transforms. The restriction leads to a set of simultaneous equations from which the polynomial coefficients can be obtained. This method provides two interesting possibilities, it can estimate the dc resistance of an electrochemical system and it can indicate whether the measured data set complies with the $\mathrm{K}-\mathrm{K}$ transforms. A clear offset between the measured and the extrapolated frequency ranges is indicative of non $\mathrm{K}-\mathrm{K}$ compliance. The adjusting procedure for the polynomials is, however, rather complex, execution times of $5 \mathrm{~min}$ on a $16 \mathrm{MHz} 386$ computer with math coprocessor have been reported [22]. A point of discussion is to what extent the dispersion of the "dominant time constant in the lower frequency domain" should be present in the measured data set. If the same requirements as presented in this paper must be met, then the method described in this paper presents at least equal accuracy at a much greater speed (seconds versus minutes).

A quite different approach to a general $\mathrm{K}-\mathrm{K}$ test of impedance data was presented by Agarwal and Orazem $[23,24]$. This method is based on modelling of the dispersion with a large number of parallel $R-$ $C$ circuits connected in series (Voigt model). $R-C$ circuits are added until the overall dispersion equals, within a certain preset error limit, the measured dispersion. As each $R-C$ circuit is $\mathrm{K}-\mathrm{K}$ transformable the entire model obeys the $\mathrm{K}-\mathrm{K}$ transform. Hence if parts of the measured dispersion (generally the end regions) fail to be modelled within the error limit then these must be considered to be corrupted. In order to keep the number of $R-C$ circuits manageable a rather large (e.g. $2 \%$ ) fit error must be accepted. So far the method has been applied successfully on a number of systems, however, it remains to be proven that it can also be applied to special cases such as e.g. an "open ended" CPE or bounded diffusion dispersion. 
Quite recently two methods based on Fourier transforms have been presented by Vander Noot [25] and Macdonald [17]. The latter method, using special multiple Fourier transforms, is able to remove systematic errors from data that does not comply with the $\mathrm{K}-\mathrm{K}$ transforms. How well these methods can be used with incomplete data is not yet clear.

As mentioned above, some electrochemical systems contain a negative differential resistance due to the negative slope of the polarisation curve at the applied polarisation. Dispersion curves of these systems do not comply with the $\mathrm{K}-\mathrm{K}$ transforms. However, quite often they can very well be modelled with an equivalent circuit [14] and thus should not be rejected solely on grounds of the $\mathrm{K}-\mathrm{K}$ transform results. When a negative resistance is found parallel to a negative capacitance (or negative CPE) then the dispersion does comply with the $\mathrm{K}-\mathrm{K}$ transformation. This type of sub-circuit is often encountered in the equivalent circuit used to model electrode reactions.

\section{Conclusions}

If an impedance spectrum can be modelled satisfactorily in a CNLS-fit procedure using an equivalent circuit then data validation by Kramers-Kronig transformation becomes superfluous. In cases where no "good fit" can be obtained it is useful to check if the data is $\mathrm{K}-\mathrm{K}$ transformable. In that case it is important to extend the frequency range far enough that the dispersion can be extrapolated at the extremes by a "single time constant" model each. Statistical noise in the transformed data is generally of the same magnitude as in the original data, hence systematic errors will not be masked in the $\mathrm{K}-\mathrm{K}$ transform pair.

Inductive effects appearing at high frequencies, due to the leads and/or instrumentation, must be removed carefully from the measured dispersion before a $\mathrm{K}-\mathrm{K}$ transform can be executed in the impedance plane. Dispersions of blocking electrodes (nonterminated CPE behaviour at low frequencies), although violating the $\mathrm{K}-\mathrm{K}$ ground rules, obey the $\mathrm{K}$ $\mathrm{K}$ transforms. Hence this type of impedance data may equally well be subjected to data validation using the $\mathrm{K}-\mathrm{K}$ transforms.

\section{References}

[1] J.R. Macdonald and L.D. Potter, Jr., Solid State Ionics 24 (1987) 61

[2] J.R. Macdonald, J. Appl. Phys. 65 (1989) 4845.

[3] J.R. Macdonald, Electrochim. Acta 35 (1990) 1483.

[4] B.A. Boukamp, Solid State Ionics 20 (1986) 31.

[5] B.A. Boukamp, Equivalent Circuit, Internal report CT89/ 214/128 (University of Twente, 1989).

[6] B.A. Boukamp, Solid State Ionics $18 / 19$ (1986) 136

[7] B.A. Boukamp, 9th Europ. Congr. on Corrosion (Royal Netherlands Industries Fair, 1989) FU-252.

[8] B.A. Boukamp, B.A. van Hassel and A.J. Burggraaf, in: Symp. on High Temperature Electrode Materials and Characterization, eds. D.D. Macdonald and A.C. Khandkar, Electrochem. Soc. Proc. Vol. 91-6 (1991) p. 44.

[9] D.D. Macdonald and M.C.H. McKubre, in: Impedance Spectroscopy, Emphasizing Solid Materials and Systems, ed. J. Ross Macdonald (Wiley, New York, 1987) pp. 260-316.

[10] R.L. Hurt and J.R. Macdonald, Solid State Ionics 20 (1986) 111.

[11] B.A. Boukamp, Solid State Ionics 11 (1984) 339.

[12] H.W. Bode, Network Analysis and Feedback Amplifier Design (Van Nostrand, New York, 1945).

[13] D.D. Macdonald and M. Urquidi-Macdonald, J. Electrochem. Soc. 132 (1985) 2316.

[14] J.A.L. Dobbelaar, The use of impedance measurements in corrosion research, Ph. D. Thesis (Delft University of Technology, 1990) p. 28.

[15] M. Urquidi-Macdonald, S. Real and D.D. Macdonald, J. Electrochem. Soc. 133 (1986) 2018.

[16] M. Urquidi-Macdonald, S. Real and D.D. Macdonald, Electrochim. Acta 35 (1990) 1559.

[17] J. Ross Macdonald, presented at the 2nd Int. Symp. on Electrochemical Impedance Spectroscopy, Santa Barbara, July 1992 (Proc. to appear in Electrochim. Acta.).

[18] J. Ross Macdonald and M.K. Brachman, Rev. Mod. Phys. 28 (1965) 393.

[19] W.H. Press, B.P. Flannery, S.A. Teukolsky, W.T. Vettering, Numerical Recipes (Cambridge University Press, Cambridge, 1986).

[20] I.D. Raistrick, C. Ho, Y-W. Hu and R.A. Huggins, J. Electroanal. Chem. 77 (1977) 319.

[21] M.E. Orazem, J.M. Esteban and O.C. Moghissi, Corrosion 47 (1991) 248.

[22] J.M. Esteban and M.E. Orazem, J. Electrochem. Soc. 138 (1991) 67

[23] P. Agarwal and M.E. Orazem, J. Electrochem. Soc. 139 (1992) 1917.

[24] P. Agarwal and M.E. Orazem, presented at the 2nd Int. Symp. on Electrochemical Impedance Spectroscopy, Santa Barbara, July 1992 (Proc. to appear in Electrochim. Acta.).

[25] T.J. Vander Noot, J. Electroanal. Chem. 322 (1992) 9. 